\title{
Bent-Bent Waveguide Coupling System
}

\author{
João Paulo N. Torres, António Baptista, Vitor Maló Machado and Ricardo A. Marques Lameirinhas
}

\section{ABSTRACT}

In this paper, the mechanism of energy transfer between two bent-bent waveguides is analyzed. Focus is done to the effect that some parameters like the refractive index of the substrate and the wavelength cause in the energy rate transfer between the waveguides. Results were validated by using the Finite Element Method (FEM).

Keywords: Bent Waveguides, Coupled Theory, Finite Element Methods.

\section{INTRODUCTION}

Bent waveguides, resonators, lasers, and other optical components are important devices in optoelectronics [1][25]. These devices are important due to their particular configuration useful for resonant applications as well as their capability to implement compact, small size devices [6]-[25].

However, the main disadvantage of the ring configuration applied to waveguides is the occurrence of the inherent curvature losses. Waveguide coupling has been studied for some decades concerning parallel waveguides [14]-[18] and non-parallel waveguides, between bent and straight optical waveguides [2], [3], [5], [19], between two non-concentric bent waveguides [20] or even between concentric ring waveguides [1], [4]. This last case, the coupling between concentric ring waveguides, is treated in the paper. This case has importance namely for couplers between ring lasers and curved passive waveguides where curved regions can be simulated by using concentric ring waveguide coupling
Published Online: January 5, 2021

ISSN: $2684-4451$

DOI :10.24018/ejphysics.2021.3.1.44

\section{João Paulo N. Torres}

Department of Electrical and Computer Engineering, Instituto Superior Técnico, Lisbon, Portugal.

Instituto de Telecomunicações, Lisbon, Portugal.

(e-mail: joaotorres@tecnico.ulisboa.pt) António Baptista

Department of Electrical and Computer Engineering, Instituto Superior Técnico, Lisbon, Portugal.

Vitor Maló Machado

Department of Electrical and Computer Engineering, Instituto Superior Técnico, Lisbon, Portugal. Instituto de Telecomunicações, Lisbon, Portugal.

Ricardo A. Marques Lameirinhas* Department of Electrical and Computer Engineering, Instituto Superior Técnico,Lisbon, Portugal.

Instituto de Telecomunicações, Lisbon, Portugal.

(e-mail: $\quad$ ricardo.lameirinhas@

tecnico.ulisboa.pt)

*Corresponding Author analysis. The coupled mode theory (CMT) [2], [3], [15][18], [20], is applied to non-parallel waveguide cases. Instead of using Cartesian co-ordinates [2], [3], [20] or conformal transformations [4], in this paper, cylindrical coordinates are used directly from the very beginning of the field problem formulation. In section II, the CMT in cylindrical co-ordinates is presented. In section III numerical results are presented and in section IV conclusions are involved. Results are compared with those obtained by using the finite element method (FEM). The FEM is adopted in the frequency domain by using a formulation for $2 \mathrm{D}$ waveguide field problems.

\section{Bent-Bent Coupling Model}

In this section the coupling model is introduced for a bent-bent waveguide structure presented in Fig. 1. 


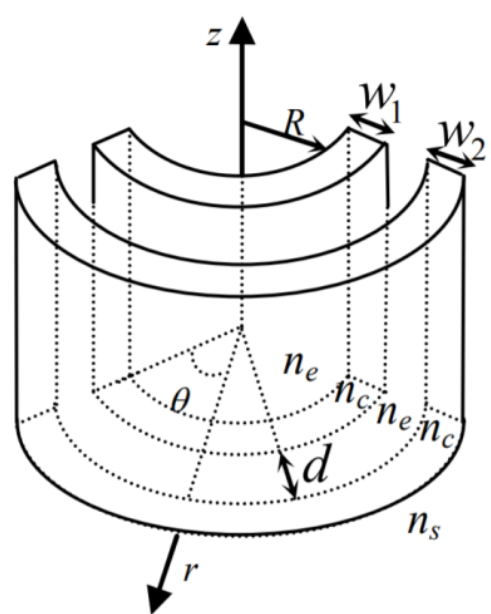

Fig. 1. Two coupled ring waveguides where $\mathrm{R}$ is the curvature radius, $w_{1}$ and $w_{2}$ are the waveguide widths, $\mathrm{d}$ is the separation distance and $n_{e}, n_{c}$ and $n_{s}$ are the refractive indices of the external region, of the waveguides and of the outer waveguide substrate respectively.

The structure is a combination of two bent waveguides coupled to each other: an inner waveguide a, and an outer waveguide b. The CMT is used where the weak coupling approximation is assumed. As a consequence, the field is obtained as a linear combination of the fundamental modes in each isolated waveguide. The complex amplitude of the axial component of the electric field (TE mode), is given by [1]:

$\bar{E}(r, \theta)=a(\theta) \overline{E_{1}^{0}}(r) e^{-j v_{1} \theta}+b(\theta) \overline{E_{2}^{0}}(r) e^{-j v_{2} \theta}$

Coefficients $a(\theta)$ and $b(\theta)$ are determined by adjusting the amplitude of the individual fields. $\overline{E_{1}^{0}}(r)$ is the field profile in guide 1 and $\overline{E_{2}^{0}}(r)$ is the field profile in waveguide 2 . To achieve the amplitudes a and $\mathrm{b}(1)$, the Lorentz reciprocity theorem is used for two electromagnetic waves. This result follows from the identity:

$$
\nabla \cdot\left(\overline{\boldsymbol{E}_{p}} \times \overline{\boldsymbol{H}_{q}^{*}}\right)+\nabla \cdot\left(\overline{\boldsymbol{E}_{q}^{*}} \times \overline{\boldsymbol{H}_{p}}\right)=-j \omega\left(\overline{\varepsilon_{p}}-\overline{\varepsilon_{q}^{*}}\right)\left(\overline{\boldsymbol{E}_{q}^{*}} \times \overline{\boldsymbol{E}_{p}}\right)
$$

Developing the first two terms, taking into account (1), the following results are obtained in cylindrical co-ordinates for $\mathrm{i}=1,2$ :

$$
\begin{aligned}
& \nabla .\left(\overline{\boldsymbol{E}} \times \overline{\boldsymbol{H}_{i}^{*}}\right)=\nabla \cdot\left(\overline{\boldsymbol{E}_{1}} \times \overline{\boldsymbol{H}_{i}^{*}}\right) \frac{1}{r} \frac{\partial a}{\partial \theta} \boldsymbol{e}_{\theta}+a \nabla \cdot\left(\overline{\boldsymbol{E}_{1}} \times \overline{\boldsymbol{H}_{i}^{*}}\right)+ \\
& \nabla .\left(\overline{\boldsymbol{E}_{2}} \times \overline{\boldsymbol{H}_{i}^{*}}\right) \frac{1}{r} \frac{\partial b}{\partial \theta} \boldsymbol{e}_{\theta}+b \nabla \cdot\left(\overline{\boldsymbol{E}_{2}} \times \overline{\boldsymbol{H}_{i}^{*}}\right) \\
& \nabla .\left(\overline{\boldsymbol{E}_{i}^{*}} \times \overline{\boldsymbol{H}}\right)=\nabla \cdot\left(\overline{\boldsymbol{E}_{i}^{*}} \times \overline{\boldsymbol{H}_{1}}\right) \frac{1}{r} \frac{\partial a}{\partial \theta} \boldsymbol{e}_{\theta}+a \nabla \cdot\left(\overline{\boldsymbol{E}_{i}^{*}} \times \overline{\boldsymbol{H}_{1}}\right)+ \\
& \nabla .\left(\overline{\boldsymbol{E}_{i}^{*}} \times \overline{\boldsymbol{H}_{2}}\right) \frac{1}{r} \frac{\partial b}{\partial \theta} \boldsymbol{e}_{\theta}+b \nabla \cdot\left(\overline{\boldsymbol{E}_{i}^{*}} \times \overline{\boldsymbol{H}_{2}}\right)
\end{aligned}
$$

Addition of expression 3 and 4 yields the result:

$$
\begin{aligned}
& \frac{\partial a}{\partial \theta}\left\{\boldsymbol{e}_{\theta} \frac{1}{r}\left(\overline{\boldsymbol{E}_{1}} \times \overline{\boldsymbol{H}_{i}^{*}}+\overline{\boldsymbol{E}_{i}^{*}} \times \overline{\boldsymbol{H}_{1}}\right)\right\}+\frac{\partial b}{\partial \theta}\left\{\boldsymbol { e } _ { \theta } \frac { 1 } { r } \left(\overline{\boldsymbol{E}_{2}} \times \overline{\boldsymbol{H}_{i}^{*}}+\right.\right. \\
& \left.\left.\overline{\boldsymbol{E}_{i}^{*}} \times \overline{\boldsymbol{H}_{2}}\right)\right\}=-j \omega\left(\bar{\varepsilon}-\overline{\varepsilon_{1}}\right)\left(\overline{\boldsymbol{E}_{i}^{*}} \times \overline{\boldsymbol{E}_{1}}\right) a-j \omega(\bar{\varepsilon}- \\
& \left.\overline{\varepsilon_{2}}\right)\left(\overline{\boldsymbol{E}_{i}^{*}} \times \overline{\boldsymbol{E}_{2}}\right) b
\end{aligned}
$$

The integration of (5) over the waveguide cross section, gives the following system of ordinary differential equations in order to determine the amplitudes a and $\mathrm{b}$ :

$\left(\begin{array}{ll}\sigma_{11} & \sigma_{12} \\ \sigma_{21} & \sigma_{22}\end{array}\right) \frac{d}{d \theta}\left(\begin{array}{l}a \\ b\end{array}\right)=\left(\begin{array}{cc}c_{11} & c \\ c_{21} & c_{22}\end{array}\right)\left(\begin{array}{l}a \\ b\end{array}\right)$

where the coefficients $\sigma_{p q}$ and $c_{q p}$ are:

$$
\begin{aligned}
& \sigma_{p q}=\int \frac{1}{r} e_{\theta} \overline{\boldsymbol{E}_{q}} \times \overline{\boldsymbol{H}_{p}^{*}}+\overline{\boldsymbol{E}_{p}^{*}} \times \overline{\boldsymbol{H}_{q}} d r \\
& c_{q p}=-j \omega \int\left(\bar{\varepsilon}-\overline{\varepsilon_{q}}\right)\left(\overline{\boldsymbol{E}_{p}^{*}} \times \overline{\boldsymbol{E}_{q}}\right) d r
\end{aligned}
$$

Expression (6) was solved by using two methods, the Runge Kutta and finite element method (FEM). Results show that both methods behave consistently.

\section{RESULTS}

In this paper, coupling between two bent waveguides are analyzed in order to understand the energy transfer mechanism in this kind of structures. The structure is composed by two waveguides: an inner waveguide where the energy is injected and an outer waveguide to where the energy is transferred. It was studied the effects on the energy transfer rate of two parameters: the wavelength for a fixed curvature radius; and, the refractive index of the outer waveguide substrate. Results were obtained considering the data presented in table 1 .

\begin{tabular}{ccccc}
\multicolumn{5}{c}{ TABLE 1: STRUCTURE PARAMETERS } \\
\hline$n_{c}$ & $n_{e}$ & $\mathrm{R}(\mu \mathrm{m})$ & $\mathrm{d}(\mu \mathrm{m})$ & $w_{1}=w_{2}(\mu \mathrm{m})$ \\
\hline 3.6 & 3.35 & 5 & 0.1 & 0.2 \\
\hline
\end{tabular}

Firstly, it was analyzed the influence caused by the wavelength on the energy transfer rate from the inner waveguide to the outer one, taking $n_{s}=3.35$. Fig. 2 illustrates the energy transfer rate from the inner waveguide to the outer one along the coordinate $\theta$. In this plot, $P_{2}$ describes the energy transfer rate being defined by $P_{2}=$ $\left|b(\theta) e^{-j v_{2} \theta}\right|^{2}$.

In all results, the mode profiles $\overline{E_{1}^{0}}(r)$ and $\overline{E_{2}^{0}}(r)$ was acquired by using two different methods: the finite element method (numerical method) [13] and a semi-analytic method [1]. These two methods show a maximum deviation of $0.1 \%$ in the results. In addition to the field profiles, the equation of coupled modes was also solved by two different methods: the Runge Kutta method and again the finite element method. The maximum deviation found between these two methods was $0.2 \%$.

Results show that the energy transfer rate has a periodic variation with theta. The differences in the period of the variation are due to a change in the ratio between the separation distance $d$ and the wavelength $\lambda$. A decrease in this ratio leads to an increase of the period of the energy transfer rate. 


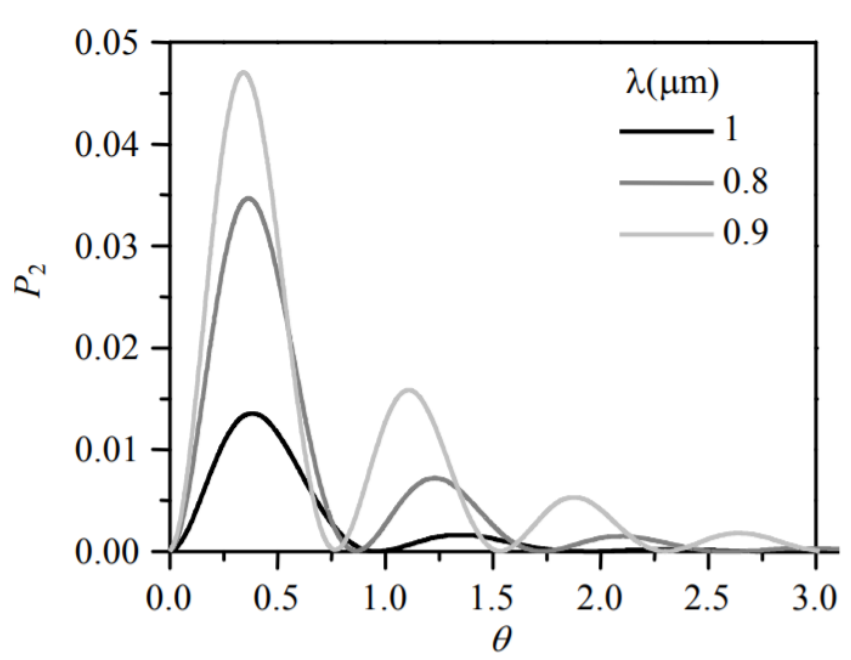

Fig. 2. Energy transfer rate from the inner waveguide to the outer one for three wavelengths values for fixed curvature radius $\mathrm{R}=5 \mu \mathrm{m}$ and separation distance between the waveguides $\mathrm{d}=0.1 \mu \mathrm{m}$.

From Fig. 2 it is possible to observe the existence of an absolute maximum for the energy transfer rate. The location of this maximum value is practically invariant with the wavelength. However, this maximum decreases in amplitude as the wavelength increases.

This effect is consequence of the decreasing of the ratio between the curvature radius and the wavelength. When the wavelength increases the curvature losses also increases. All the curves in Fig. 2 show a decrease with theta of the energy rate transfer from the inner to the outer guide. This effect is, for a constant radius, a consequence of the curvature losses.

Secondly, the wavelength was fixed $(0.9 \mu \mathrm{m})$ and the refractive index of the outer waveguide substrate was used as a parameter.

The calculated energy transfer rate from the inner waveguide to the outer one also shows an absolute maximum value. However, the position of the maximum is sensitive to the substrate refractive index of the outer waveguide. An interesting consequence is the increase of the average energy transfer rate as the refractive index of the outer waveguide substrate decreases (Fig. 3).

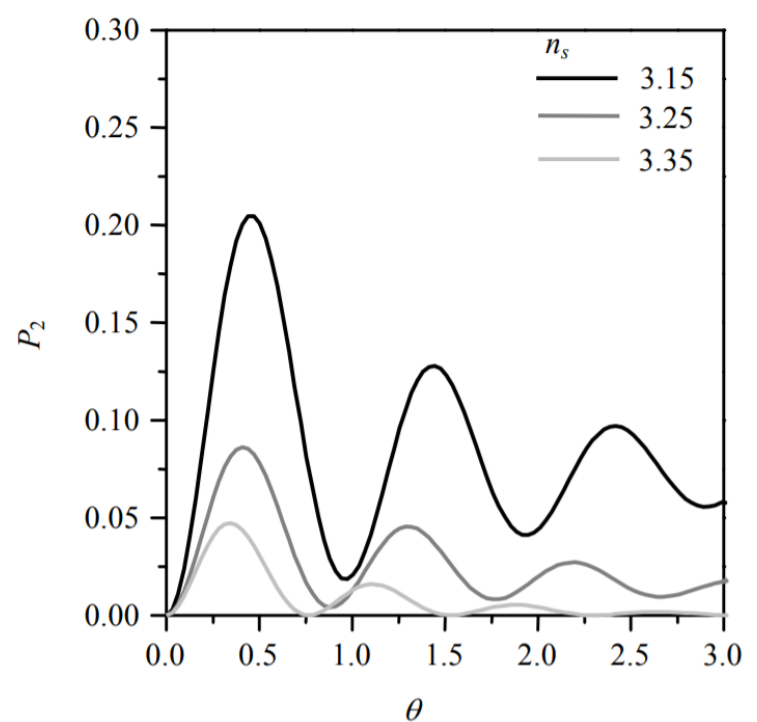

Fig. 3. Energy transfer rate, with $\lambda=0.9 \mu \mathrm{m}$, from the inner waveguide to the outer one taking the refractive index of the outer waveguide substrate as a parameter.
The analysis of the energy propagated along the azimuthal coordinate (Fig. 4), allows to observe that a decrease of the outer waveguide substrate refractive index leads to an increase in the energy transfer rate from the inner waveguide to the outer one. The energy transfer rate from the external waveguide to the internal one is much smaller than the corresponding transfer in the opposite direction. This situation is different from what occurs in straight waveguides where the transfer rate is equal in both directions for similar waveguides.

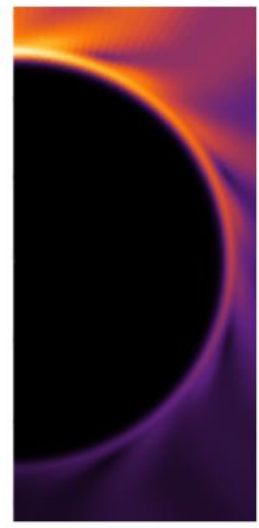

a)

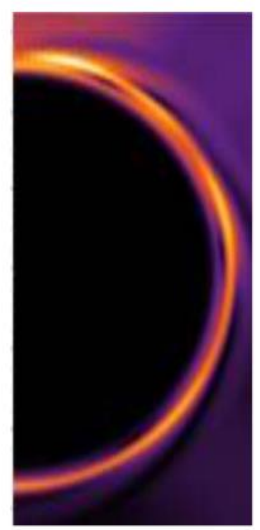

b)
Fig. 4. Energy transfer along the azimuthal coordinate with $\lambda=0.9 \mu \mathrm{m}$ for two values of the outer waveguide substrate refractive: a) $\left.n_{\mathrm{s}}=3.35 ; \mathrm{b}\right) n_{\mathrm{s}}=3.15$.

\section{CONCLUSION}

In this paper it was studied the coupling in bent-bent waveguide systems in order to understand the mechanism of energy transfer between concentric waveguides.

It was analyzed the effect caused by the wavelength and the outer waveguide substrate refractive index of the outer waveguide. We observed the existence of a maximum value for the energy transfer rate from the inner waveguide to the outer one. These maximum values are located practically in the same azimuthal position for the different wavelength values but change their position with the outer waveguide substrate refractive index.

A periodic oscillation of the energy transfer rate can be observed between the inner waveguide to the outer one.

\section{ACKNOWLEDGMENT}

This work was supported in part by FCT/MCTES through national funds and in part by cofounded EU funds under Project UIDB/EEA/50008/2020.

\section{REFERENCES}

[1] João Torres, António Baptista, and Victor Maló Machado, "Coupling analysis in concentric ring waveguides". Journal of lightwave technology 31.13: 2140-2145, 2013.

[2] R. Stoffer, K. R. Hiremath, and M. Hammer, "Comparison of Coupled Mode Theory and FDT Simulations Of Coupling between Bent and Straigth Optical Waveguides." International School of Quantum Eletronics, 39yh Course: Erice, Italy, 18-25, Oct. 2004.

[3] K.R. Hiremath, R. Stoffer and M. Hammer, "Coupled Mode Theory and FDT Simulations of the Coupling Between Bent and Staigth 
Optical Waveguides", Proceedings Symposium IEEE/Leos Benelux Chapter, 2003 [4] L. Djaloshinski and M. Orenstein, "Disk and Ring Microcavity Lasers and Their concentric Coupling", IEEE J. Quantum Electronics, vol. 35, no. 5, pp. 737-744, May 1999.

[4] J. Ctyroký, I. Richter and M. Sinor, "Dual Resonance in a Waveguidecouple ring microresonator", Springer Optical and Quantum Electronics, vol. 38, pp. 781-797, 2006.

[5] J. Torres, A. Baptista, V. M. Machado, "Analysis of dielectric ring slab waveguides with interband absortion", EUROCON 2011 proceedings, Lisbon, 27-28 April 2011.

[6] J. Torres, A. Baptista, V. M. Machado, "Analysis of dielectric optical ring slab waveguides with a layred refractive index", 18th Telecomunication Forum, TELFOR 2010 proceedings, Belgrade, Serbia, 23-25 Nov. 2010.

[7] J. Torres, A. Baptista, V. M- Machado, "Curvature Losses Of Slab Waveguides Using Analytical and FEM Analysis" ODEF10 Proceedings, Japan, 2010.

[8] E. A. Maracatili, "Bends in Optical Dielectric Guides", The Bell System Technical Journal, pp. 2103-2160 Sept. 1969.

[9] D. Marcuse, "Bending losses of the Asymmetric Slab waveguide", Bell System Technical Journal, vol. 50, no. 8, Oct. 1971

[10] M. Heiblum, J. H. Harris, "Analysis of Curved Optical Waveguides by Conformal Transformation”, IEEE J. Quantum Electronics, vol. QE-11, no. 2 Feb. 1975.

[11] K. R. Hiremath, M. Hammer, R. Stoffer, L. Prkna and J. Ctyroky, "Analytic approach to dielectric bent slab waveguides", Springer Optical and Quantum Electronics, vo. 37, pp. 37-61, 2005.

[12] T. Yamamoto and M. Koshiba, "Numerical Analysis of Curvature Loss in Optical Waveguides by the Finite-Element Method", vol. 11, no. 10, pp. 1579-1583, Oct. 1993.

[13] D. Marcuse, The coupling of degenerate modes in tow parallel dielectric waveguides, The Bell System Technical Journal, vol. 50, no. 6, pp. 1791-1815, July-August 1971.

[14] A. Hardy and W. Streifer, "Coupled Mode Theory of Parallel Waveguides", IEEE, J. Ligthwave Tecnhology, vol. LT-3, no. 5, pp. 1135-1146, Oct 1985.

[15] H. A. Haus, W. P. Haung, S. Kawakami and N. A. Whitaker, "CoupledMode Theory of Optical Waveguides", IEEE J. Ligthwave Tecnhology, vol. LT-5, no. 1, pp. 16-23, Jan 1987.

[16] B. E Little and W. P. Huang, "Coupled-mode theory for optical waveguides", Progress In Electromagnetics Research, Pier 10, pp. 217- 270, 1995.

[17] H. A. Haus, "Waves and Fields in Optoelectronics", Prentice-Hall Series in Solid State Physical Electronics, Nick Holopvak Jr. Editor.

[18] A. Yariv, "Critical Coupling and Its Control Optical WaveguideRing Resonator Systems", vol. 14, no. 4, April 2002.

[19] M. A. McHenry and D. C. Chang, "Coupled Mode Theory of Tow Non-parallel Dielectric Waveguides", Scientific Report no. 73 Electromagnetics Laboratory Department of Electrical Engineering University of Colorado Boulder, Colorado.

[20] R. A. Marques Lameirinhas, J. P. N. Torres and A. Baptista, "The Influence of Structure Parameters on Nanoantennas' Optical Response". Chemosensors, 8, 42, 2020.

[21] R. A. Marques Lameirinhas, J. P. N. Torres and A. Baptista, "Sensors Based on Nanoantennas: Fundamentals". European Journal of Applied Physics, 2, 3, 2020.

[22] R. A. Marques Lameirinhas, J. P. N. Torres and A. Baptista, "A Sensor Based on Nanoantennas". Applied Sciences 10.19: 6837, 2020.

[23] T. Bonnal, et al, "How to determine the complex refractive index from infrared reflectance spectroscopy?". SN Applied Sciences 2.12: $1-9,2020$.

[24] Joao Paulo N. Torres, V. Machado, and Antonio Baptista, “A New Hybrid Finite Element Method: Electromagnetic Propagation in Bent Waveguides”. IPhoJ 12.1: 2966256, 2020. 